\title{
Shake Table Statistical Validation of Finite Element Models of Rocking Structures
}

\section{Conference Paper}

\section{Author(s):}

Katsamakas, Antonios; Vassiliou, Michalis F.

Publication date:

2021

Permanent link:

https://doi.org/10.3929/ethz-b-000476573

Rights / license:

In Copyright - Non-Commercial Use Permitted

Originally published in:

https://doi.org/10.5592/CO/1CroCEE.2021.246 


\title{
SHAKE TABLE STATISTICAL VALIDATION OF FINITE ELEMENT MODELS OF ROCKING STRUCTURES
}

\author{
Antonios A. Katsamakas ${ }^{(1)}$, Michalis F. Vassiliou ${ }^{(2)}$ \\ (1) Ph.D. Candidate, Chair of Seismic Design and Analysis, IBK, ETH Zurich \\ (2) Assistant Professor, Chair of Seismic Design and Analysis, IBK, ETH Zurich
}

\begin{abstract}
This paper presents a three-dimensional finite element (FE) modeling approach for predicting the response of rocking columns. The model is validated against experimental results, which involved testing three different cylindrical columns with different slenderness ratios under a set of 100 bidirectional ground motions. Each column was free-standing and allowed to slide and rock in all directions. Since the developed stresses in the columns were low, all columns were modeled as rigid.

The contact surface was simulated using Coulomb friction for the tangential behavior and stiff contact for the normal direction. Two energy dissipation mechanisms were modeled; friction and radiation damping. Inherent numerical damping, as well as Rayleigh damping, were set to zero, with this approach complying to the physical problem.

Rocking is characterized as a chaotic and unpredictable problem, with tests being non-repeatable. Therefore, this study employs a statistical approach to validate the numerical results, using the cumulative distribution function (CDF) for the main response quantity (i.e., maximum top displacement of the column). It was proved that the model performs poorly in the deterministic validation but demonstrates satisfying agreement with the experimental results when validated statistically.

The influence of the main modeling parameters, meaning the friction coefficient and the radiation damping properties, was assessed through an extensive sensitivity analysis using non-linear time-history analyses. A small change of the value of these parameters leads to a different individual rocking oscillation but only smoothly influences the statistical response.
\end{abstract}

Keywords: rocking columns; finite element modeling; statistical validation; sensitivity analysis; free-standing equipment

\section{Introduction}

Rocking structures are the ones that are allowed to uplift when subjected to dynamic excitation. Uplifting occurs when the ratio of the acceleration of the excitation $\left(\ddot{u}_{g}\right)$ divided by the gravity acceleration $(g)$ is larger than the slenderness of the block $(\alpha)$ and the sliding surface is sufficiently rough. This uplifting effect acts as a fuse, limiting the inertial forces transmitted to the superstructure. After uplift, a rocking oscillator demonstrates negative stiffness, making the description of such systems significantly different than the conventional ones.

The first analytical study of this phenomenon is dated back to 1885 [1,2]. However, rocking structures have been systematically studied after 1963, when Housner published his seminal paper entitled "The behavior of inverted pendulum structures during earthquakes" where two main properties of the rocking structures were elucidated: i) out of two geometrically similar rocking blocks (same $\alpha$ ) the larger one (larger $R$ ) can survive an excitation which will topple the smaller one ii) longer period ground motions have a higher overturning potential [3].

The rocking oscillator has been used to describe the dynamic behavior of free-standing equipment [47], masonry structures [8-14] and ancient temples [15-19]. Rocking is also a promising seismic response modification technique, both for bridges and buildings, with limited practical applications in the former USSR and New Zealand [20,21]. Applications in buildings may comprise a soft-rocking-story mechanism [22-24], or a rocking wall [25, 26], whereas in bridges, rocking piers [27-35]. Several analytical studies investigated the response of rocking structures combined with external dampers or 
restraining tendons [36-38]. The influence of the flexibility of the rocking body was also studied, both analytically and experimentally [39-42].

The analytical model proposed by Housner describes the planar rocking response of a rigid body when subjected to one-directional excitation. However, real rocking structures are subjected to bidirectional (or three-directional when the vertical acceleration is considered) excitation [43-46]. Under these conditions, an unanchored body may rock, uplift, translate with the ground, and/or wobble. When it is not restrained, it may also slide out of its initial position [47].

This study aims at developing a practical three-dimensional finite element model to predict the response of free-standing cylindrical rocking columns. The validity of the proposed model is assessed by statistically comparing numerical and experimental results. The experimental results comprise 100 shake table tests, using three cylindrical steel columns with varying slenderness. The number of tests performed is large enough to allow such a statistical comparison. The specimens were subjected to twodimensional excitation, and they were free to slide, rock and wobble in all directions. As the columns are free to slide and wobble out of their original position, they serve for validation of numerical models used for the description of the seismic behavior of equipment, rather than of structures that use rocking as a seismic isolation strategy.

\section{Statistical validation}

Rocking is often characterized as "chaotic", in the sense that the response of rocking objects is sensitive to the initial conditions, often making tests non-repeatable. Therefore, validating numerical models in a deterministic way does not even make sense.

Bachmann et al. [48] and Del Guidice et al. [49, 50] claimed that validating a numerical model using a single ground motion is a sufficient but not necessary validation procedure. The seismic response is inherently stochastic since the excitation is stochastic. Therefore, a statistical (and not a deterministic) validation of the numerical model is proposed. During this statistical validation, the statistical distributions of the main response quantities of the model and the experiments are compared. This procedure requires an experimental benchmark dataset, where the same (or identical specimens) are excited by an ensemble of ground motions. Afterwards, a numerical model is used to create another dataset, using the same ensemble of excitations. The validity of the numerical model is assessed by comparing the Cumulative Distribution Function (CDF) of these two datasets for the same response quantity (i.e., maximum top displacement). This validation test is weaker (and easier to pass), yet sufficient for earthquake engineering applications.

It is worth mentioning that this concept was first proposed by Yim, Chopra and Penzien who used it to compare different types of analyses (e.g., a simplified compared to a more refined) [51].

\section{Numerical studies of rocking structures}

During the last decade, both FEM and DEM numerical models were developed to predict the rocking problem [52-55]. A recent blind prediction contest organized by ETH Zurich, the University of Bristol and the Pacific Earthquake Engineering Research (PEER) Center, shed light on the efficiency of numerical models used to describe the statistical response of a rocking podium structure [56]. Unlike the tests discussed in this paper, the tests of [56] concerned a rocking podium structure that was restrained not to slide or wobble out of its original position. Thirteen contestants participated, using FEM, DEM, and analytical rigid-body models [57-58]. One of the important outcomes of this contest is that there is no basis for recommending FEM or DEM to model the response of wobbling structures; the accuracy of these models depends on the modeling assumptions. Moreover, it was proven that even though the winning models accurately captured the Cumulative Distribution Function of the maxima of the responses to each set of excitations, they were unable to accurately predict the response to each individual ground motion separately. 


\section{Experimental procedure}

This section briefly presents an experimental investigation designed at ETH Zurich and carried out at EQUALS Lab, University of Bristol [59]. This investigation includes 115 shake table tests of cylindrical free-standing rocking bodies, which were free to slide and rock in all directions. More details about the tests can be found in [59]. The results of these experiments were used as a benchmark database to assess the efficiency of the numerical model proposed in the present study.

The rocking specimens were not chosen to represent specific free-standing rocking equipment but a class of free-standing rocking bodies. They were designed to remain elastic after each test, so they could be excited with a large number of earthquake excitations to create a database suitable for a statistical validation. The specimens were made of round steel pipes, with different dimensions and slenderness (Figure 1).

The rocking response was induced by a di-directional dynamic excitation using a shake table. The applied ground motions were synthesized using a spectral version of the Rezaeian and Der Kiureghian stochastic ground motion model [60-61]. The 1989 Loma Prieta UCSC Lick Observatory ground motion record was used as a seed ground motion to generate an ensemble of 100 ground motions. The ground motions were scaled, with the frequency of ground motions increased by 2 without changing the amplitude. Therefore, in the prototype scale, the columns are 4 times larger.

\section{Numerical model}

The general-purpose finite element software ABAQUS [62] was utilized to create and solve the model. The model comprised the cylindrical rocking bodies, the moving flat base (which simulates the shake table), and a spring-dashpot system below the flat base to simulate the vertical stiffness of the shake table and the radiation/impact damping mechanism [63] (Figure 1, Left). All rotations of the base were fixed. The base moved parallel to $\mathrm{x}$ and $\mathrm{y}$ axis applying the ground motion. On the vertical axis ( $\mathrm{z}$ axis), the base was supported by the spring-dashpot system.

A uniform mesh with a size of $5 \mathrm{~mm}$ was utilized in all analyses. This mesh size is considered adequate to avoid mesh-related errors. A 4-node 3D rigid quadrilateral finite element was used both for the rocking column and the flat base. The motion of the specimens was monitored with a reference point at the top of the column. The contact surface was simulated using Coulomb friction for the tangential behavior and ABAQUS stiff contact [62] for the normal direction. An explicit scheme with a fixed time increment of $10^{-6} \mathrm{sec}$ was used in all cases.

The developed numerical model considers two main damping mechanisms; friction and radiation/impact damping. Inherent Rayleigh damping is set to zero since this energy dissipation mechanism is inconsistent with the physical problem. Energy dissipation through friction is considered through the friction coefficient, whereas radiation damping through the utilized dashpot. Neither the friction nor the dashpot coefficient was not known a priori; therefore, the influence of these parameters was assessed through an extensive parametric analysis employing non-linear time history analysis.
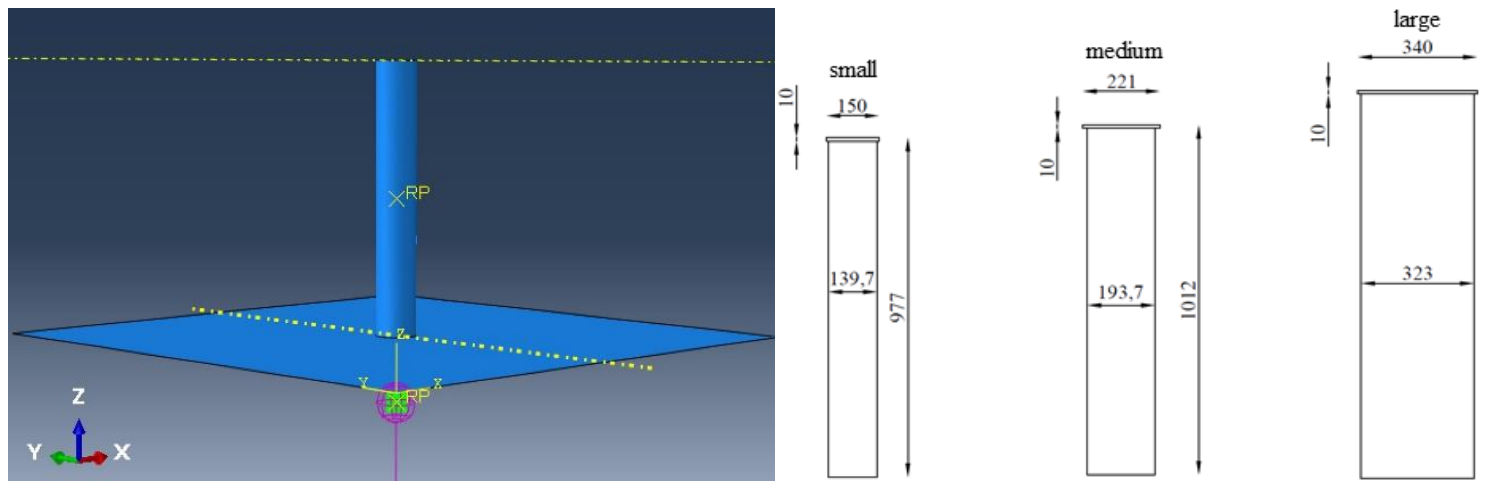

Figure 1. Left) Numerical model in ABAQUS. Right) Dimensions (in mm) of the tested specimens 


\section{Results}

\subsection{Deterministic comparison}

Figures 2 and 3 present a comparison between experimental and numerical results for the different values of the critical modeling parameters (i.e., friction and dashpot coefficient). The investigated response parameter is the maximum displacement $\left(u_{\max }\right)$ at the top of the rocking column, measured in meters. In the following plots (Figures 2,3), "OT" denotes overturning of the specimen.

It is evident that the numerical results are moderately correlated to the experimental ones. Moreover, similarly to previous studies, the numerical model often fails to predict overturning. However, the relevant question in earthquake engineering is not whether the model is accurate but whether it is biased and whether it induces more uncertainty than the ground motion one.

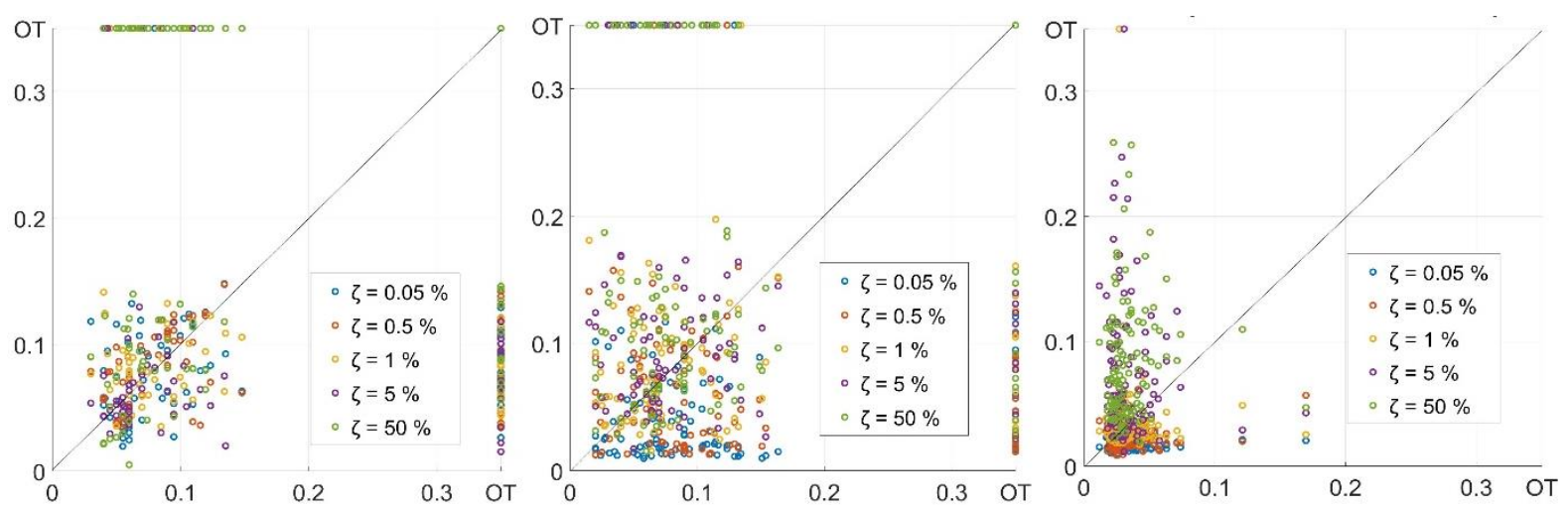

Figure 2. Deterministic comparison for experimental and numerical results of the columns. Sensitivity Analysis for dashpot coefficient Left) Small column, Middle) Medium column, Right) Large column
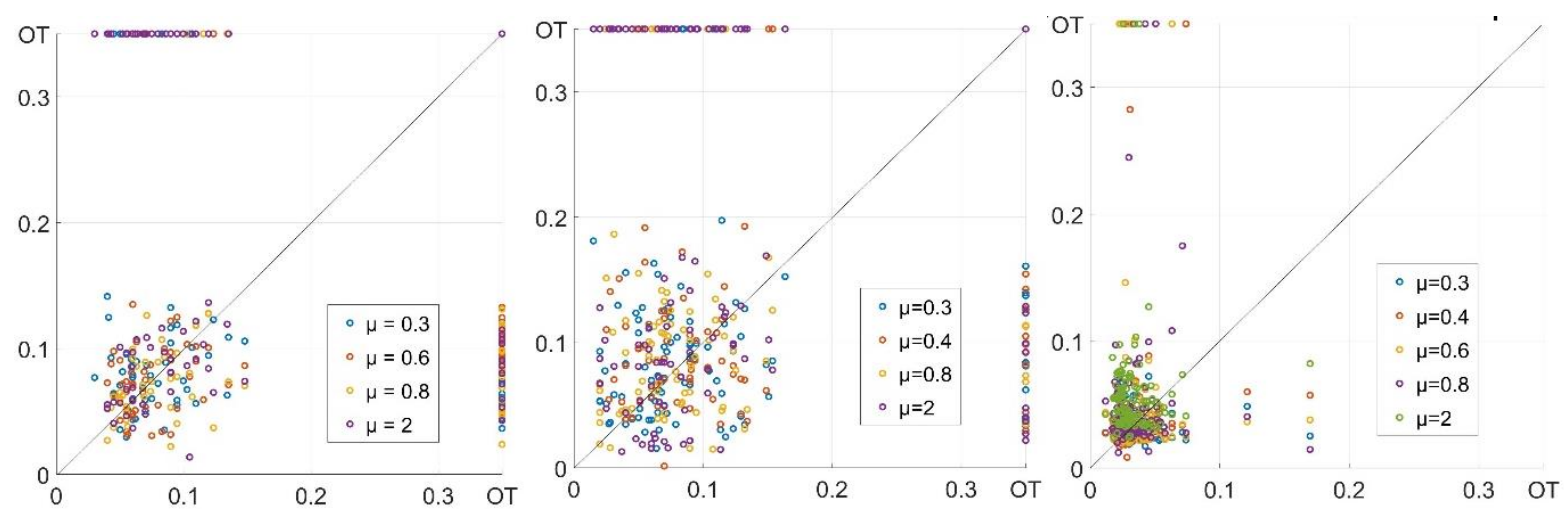

Figure 3. Deterministic comparison for experimental and numerical results of the columns. Sensitivity analysis for friction coefficient Left) Small column, Middle) Medium column, Right) Large column

\subsection{Statistical comparison}

When the numerical results are statistically assessed, clear trends emerge, similarly to what was observed for the planar rocking model by Yim et al [50] as early as in 1980 (Figures 4,5). In general, a high friction coefficient leads to higher maximum displacements of the rocking column (Figure 5). A high friction coefficient means that it is harder for the column to slide. Therefore, the transmitted inertia forces are higher, leading to uplift and larger rocking displacements.

The influence of radiation damping is also important, especially when high values are used. The CDF curves show that a high value of radiation damping makes the model conservative (Figure 5). 

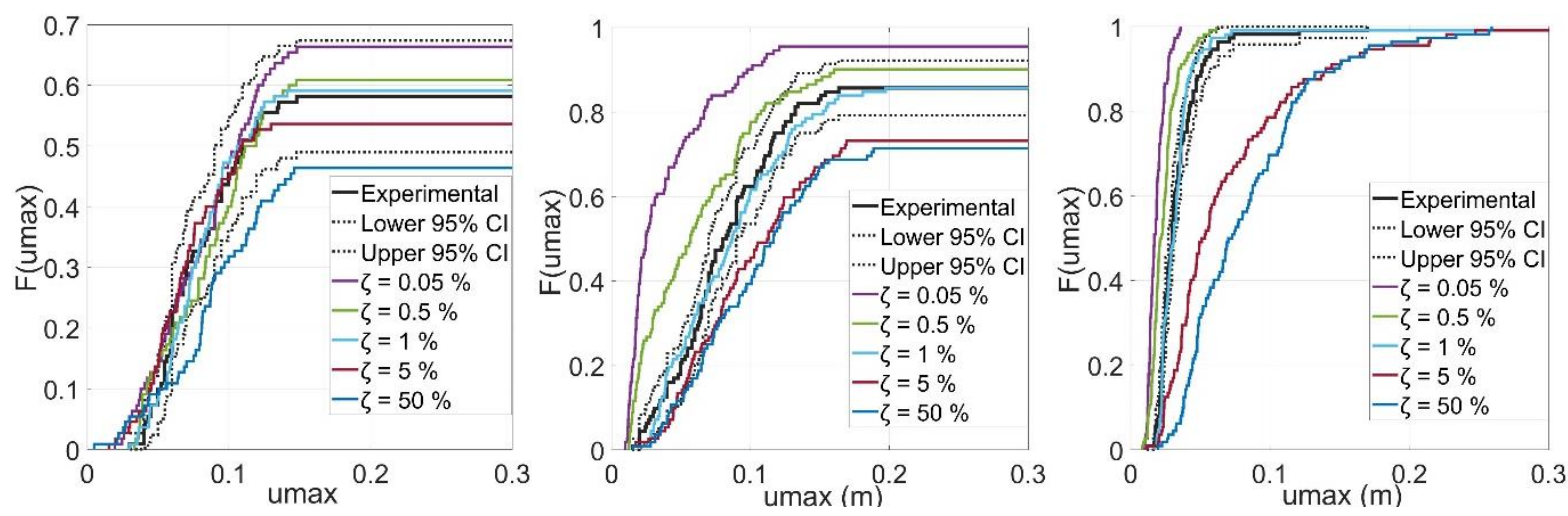

Figure 4. Statistical comparison for experimental and numerical results of the columns. Sensitivity Analysis for dashpot coefficient Left) Small column, Middle) Medium column, Right) Large column
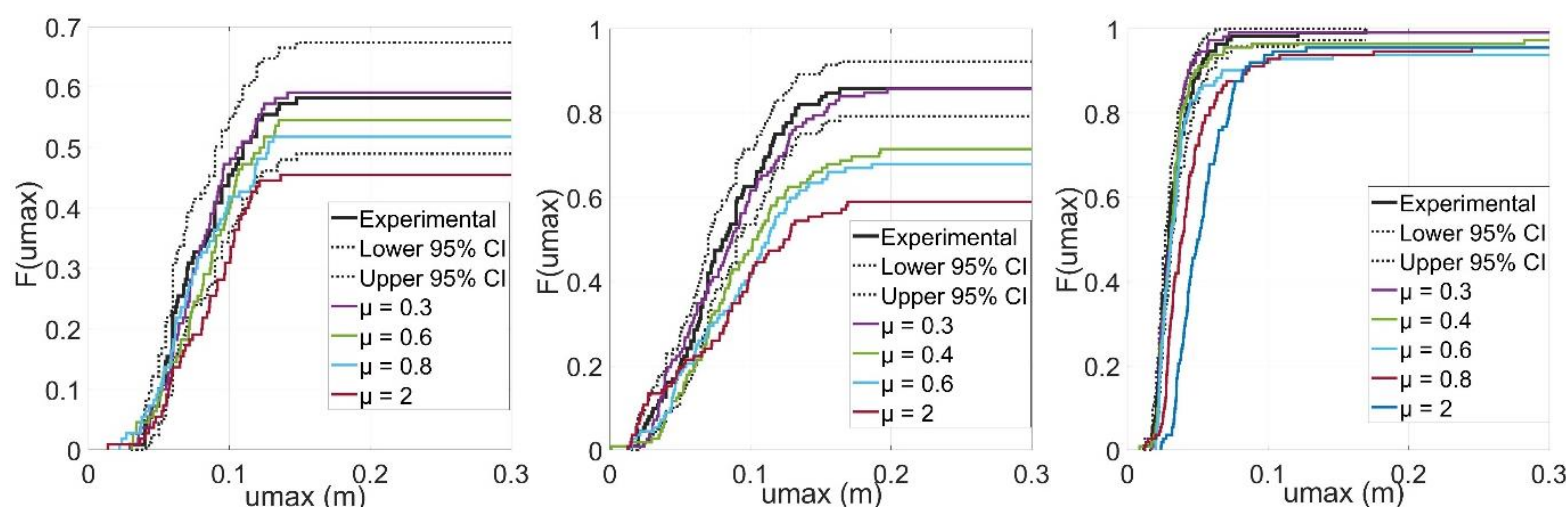

Figure 5. Statistical comparison for experimental and numerical results of the columns. Sensitivity Analysis for friction coefficient Left) Small column, Middle) Medium column, Right) Large column

\section{Conclusions}

The presented numerical model simulates the response of free-standing cylindrical columns. These columns are free to slide and rock in all directions, with their dimensions. It is shown that the model performs poorly when it is assessed base on its ability to predict the maximum to an individual ground motion. However, it performs well, when it is evaluated based on its ability to predict the CDF of the maxima of the responses to a set of ground motions.

The friction coefficient between the rocking block and the supporting surface and the dashpot used to model radiation damping were varied numerically and their influence was assessed with a large number of non-linear time-history analyses. These parameters influence moderately the statistical response of the rocking bodies. The CDF curves show that an increase of the friction or the dashpot coefficient amplifies uplifting and leads to larger maximum rocking displacements, thus making the model more conservative. A friction coefficient equal to $\mu=0.3$ and a critical damping fraction equal to $\zeta=1 \%$ leads to the optimal match between experimental and numerical results.

\section{References}

[1] Milne, J. (1885): Seismic experiments. Trans. Seismol. Soc. Jpn., 8, 1-82

[2] Makris, N. (2014): A half-century of rocking isolation. Earthquakes and Structures, 7(6), 1187-1221. https://doi.org/10.12989/EAS.2014.7.6.1187

[3] Housner, GW. (1963): The behavior of inverted pendulum structures during earthquakes. Bull Seismol Soc Am. 53(2): 403- 417. 
[4] Konstantinidis, D., Makris, N. (2009): Experimental and analytical studies on the response of free-standing laboratory equipment to earthquake shaking. Earthquake Engng Struct. Dyn. 38:827-848. DOI: 10.1002/eqe.871

[5] Konstantinidis, D., Makris, N. (2010): Experimental and analytical studies on the response of 1/4-scale models of free-standing laboratory equipment subjected to strong earthquake shaking. Bull Earthquake Eng. 8:14571477 DOI 10.1007/s10518-010-9192-8

[6] Di Sarno, L., Magliulo, G., D'Angela, D., Cosenza, E. (2019): Experimental assessment of the seismic performance of hospital cabinets using shake table testing. Earthq Eng Struct Dyn. 48(1):103-123.

[7] D'Angela, D., Magliulo, G., \& Cosenza, E. (2021). Towards a reliable seismic assessment of rocking components. Engineering Structures, 230, 111673.

[8] Stefanou, I., Psycharis, I., Georgopoulos, IO. (2011): Dynamic response of reinforced masonry columns in classical monuments. Construct Build Mater 25(12):4325-4337.

[9] DeJong MJ. (2012): Seismic response of stone masonry spires: analytical modeling. Eng Struct. 40:556 565.

[10] Tondelli M, Beyer K, DeJong M. (2016): Influence of boundary conditions on the out-of-plane response of brick masonry walls in buildings with RC slabs. Earthq Eng Struct Dynam. 45(8):1337 - 1356.

[11]Casapulla, C., Giresini, L., Lourenço, PB. (2017): Rocking and kinematic approaches for rigid block analysis of masonry walls: state of the art and recent developments. Buildings. 7(3):69

[12] Kalliontzis D, Schultz AE. (2017): Characterizing the in-plane rocking response of masonry walls with unbonded posttensioning. J Struct Eng.143(9):04017110.

[13] Mehrotra A, DeJong MJ. (2018): The influence of interface geometry, stiffness, and crushing on the dynamic response of masonry collapse mechanisms. Earthq Eng Struct Dynam.47(13):2661-2681.

[14] Mouzakis HP, Psycharis IN, Papastamatiou DY, Carydis PG, Papantonopoulos C, Zambas C. (2002): Experimental investigation of the earthquake response of a model of a marble classical column. Earthq Eng Struct Dynam.31(9):1681 - 1698.

[15]Funari, M. F., Spadea, S., Lonetti, P., Fabbrocino, F., \& Luciano, R. (2020). Visual programming for structural assessment of out-of-plane mechanisms in historic masonry structures. Journal of Building Engineering, 31, 101425.

[16] Papantonopoulos C, Psycharis IN, Papastamatiou DY, Lemos JV, Mouzakis HP. (2002): Numerical prediction of the earthquake response of classical columns using the distinct element method. Earthq Eng Struct Dynam. 31(9):1699-1717.

[17] Vassiliou MF, Makris N. (2012): Analysis of the rocking response of rigid blocks standing free on a seismically isolated base. Earthq Eng Struct Dynam. 41(2):177-196.

[18] Drosos, V.A., Anastasopoulos, I. (2015): Experimental investigation of the seismic response of classical temple columns. Bull Earthquake Eng 13, 299-310. https://doi.org/10.1007/s10518-014-9608-y

[19] Drosos V, Anastasopoulos I (2014): Shaking table testing of multi-drum columns and portals. Earthquake Engineering \& Structural Dynamics, 43(11):1703-1723.

[20]Reggiani Manzo, N, Vassiliou, MF. Displacement-based analysis and design of rocking structures. Earthquake Engng Struct Dyn. 2019; 48: 1613- 1629. https://doi.org/10.1002/eqe.3217

[21] Reggiani Manzo, N, Vassiliou, MF. (2021): Simplified analysis of bilinear elastic systems exhibiting negative stiffness behavior. Earthquake Engng Struct Dyn. 2021; 50: 580- 600. https://doi.org/10.1002/eqe.3347

[22] Bantilas, KE., Kavvadias, IE., Vasiliadis, LK. (2020): Seismic response of elastic multidegree of freedom oscillators placed on the top of rocking storey. Earthquake Engng Struct Dyn.1- 19. https://doi.org/10.1002/eqe.3400

[23]Bachmann, JA., Vassiliou, MF., Stojadinović, B. (2017): Dynamics of rocking podium structures. Earthquake Engng Struct. Dyn., 46: 2499- 2517. doi: 10.1002/eqe.2915. 
[24] Bachmann, JA, Vassiliou, MF, Stojadinovic, B. (2019): Rolling and rocking of rigid uplifting structures. Earthquake Engng Struct Dyn. 2019 48: 1556- 1574. https://doi.org/10.1002/eqe.3213

[25] Makris, N., Aghagholizadeh, M. (2017): The dynamics of an elastic structure coupled with a rocking wall. Earthquake Engng Struct. Dyn., 46: 945-962. doi: 10.1002/eqe.2838.

[26] Di Egidio, A., Pagliaro, S., Fabrizio, C., \& de Leo, A. M. (2020). Seismic performance of frame structures coupled with an external rocking wall. Engineering Structures, 224, 111207.

[27] Makris, N., Vassiliou, MF. (2013): Planar rocking response and stability analysis of an array of free-standing columns capped with a freely supported rigid beam. Earthq Eng Struct Dyn. 42(3): 431- 449.

[28] Makris, N., Vassiliou, MF. (2012): Are some top-heavy structures more stable? Journal of Structural Engineering. https://doi.org/10.1061/(ASCE)ST.1943-541X.0000933

[29] Makris, N., Vassiliou, MF. (2014): Dynamics of the rocking frame with vertical restrainers. J Struct Eng. 141(10):04014245.

[30] Dimitrakopoulos EG, Giouvanidis AI. (2015): Seismic response analysis of the planar rocking frame. J Eng Mech. 2015; 141(7):04015003.

[31] Giouvanidis AI, Dimitrakopoulos EG. (2017): Seismic performance of rocking frames with flag-shaped hysteretic behavior. J Eng Mech. 143(5):04017008.

[32] Thomaidis, IM., Kappos, AJ., Camara, A. (2020): Dynamics and seismic performance of rocking bridges accounting for the abutment-backfill contribution. Earthquake Engng Struct Dyn. 49: 1161-1179. https://doi.org/10.1002/eqe.3283

[33] Giouvanidis, A. I., \& Dong, Y. (2020). Seismic loss and resilience assessment of single-column rocking bridges. Bulletin of earthquake engineering, 18, 4481-4513.

[34] Kashani, M. M., Gonzalez-Buelga, A., Thayalan, R. P., Thomas, A. R., \& Alexander, N. A. (2018). Experimental investigation of a novel class of self-centring spinal rocking column. Journal of Sound and Vibration, 437, 308-324.

[35] Thiers-Moggia, R., \& Málaga-Chuquitaype, C. (2020). Dynamic response of post-tensioned rocking structures with inerters. International Journal of Mechanical Sciences, 187, 105927.

[36] Makris, N., Vassiliou, MF. (2015): Dynamics of the rocking frame with vertical restrainers. Journal of Structural Engineering https://doi.org/10.1061/(ASCE)ST.1943-541X.0001231

[37] Vassiliou, MF., Makris, N. (2015): Dynamics of the vertically restrained rocking column. Journal of Engineering Mechanics https://doi.org/10.1061/(ASCE)EM.1943-7889.0000953

[38] Makris, N., Aghagholizadeh M. (2019): Effect of supplemental hysteretic and viscous damping on rocking response of free-standing columns. Journal of Engineering Mechanics https://doi.org/10.1061/(ASCE)EM.1943-7889.0001596

[39] Acikgoz, S., DeJong, M. (2012): The interaction of elasticity and rocking in flexible structures allowed to uplift Earthquake Engng Struct. Dyn. 41:2177-2194 DOI: 10.1002/eqe.2181

[40] Vassiliou, MF., Mackie KR., Stojadinović B. (2014): Dynamic response analysis of solitary flexible rocking bodies: modeling and behavior under pulse-like ground excitation Earthquake Engng Struct. Dyn. 43:14631481 DOI: $10.1002 /$ eqe. 2406

[41] Vassiliou, MF., Truniger, R., Stojadinović, B. (2015): An analytical model of a deformable cantiléver structure rocking on a rigid surface: development and verification Earthquake Engng Struct. Dyn. 44:27752794 DOI: $10.1002 /$ eqe. 2608

[42] Truniger, R., Vassiliou, MF., Stojadinović, B. (2015): An analytical model of a deformable cantiléver structure rocking on a rigid surface: experimental validation Earthquake Engng Struct. Dyn 44:2795-2815 DOI: $10.1002 /$ eqe. 2609

[43] Chatzis, MN., Smyth, AW. (2012): Modeling of the 3D rocking problem. International Journal of Non-linear Mechanics. 47(4):85-98.

[44] Chatzis MN, Smyth AW. (2012): Three-dimensional dynamics of a rigid body with wheels on a moving base. Journal of Engineering Mechanics 2012; 139(4):496-511. 
[45] Vassiliou, MF., Burger, S., Egger, M., Bachmann, J., Broccardo, M., Stojadinović, B. (2017): The threedimensional behavior of inverted pendulum cylindrical structures during earthquakes. Earthquake Engng Struct. Dyn., 46: 2261-2280. doi: 10.1002/eqe.2903.

[46] Vassiliou, MF. (2018): Seismic response of a wobbling 3D frame. Earthquake Engng Struct Dyn. 47: 1212- 1228. https://doi.org/10.1002/eqe.3013

[47]Bao, Y., \& Konstantinidis, D. (2020). Dynamics of a sliding-rocking block considering impact with an adjacent wall. Earthquake Engineering \& Structural Dynamics, 49(5), 498-523.

[48] Bachmann, J.A., Strand, M., Vassiliou, M.F., Broccardo, M., Stojadinović, B. (2017): Is rocking motion predictable? Earthquake Engng Struct Dyn.47: 535-552. DOI: 10.1002/eqe.2978

[49]Del Guidice, L., Vassiliou, MF. (2020): Mechanical properties of 3D printed material with binder jet technology and potential applications of additive manufacturing in seismic testing of structures. Additive Manufacturing. https://doi.org/10.1016/j.addma.2020.101714

[50]Del Giudice, L., Wrobel, R., Leinenbach, C., \& Vassiliou, M. F. (2020). Static testing of additively manufactured microreinforced concrete specimens for statistical structural model validation at a small scale. In 8th International Conference on Advances in Experimental Structural Engineering (8AESE).

[51] Yim, C.S., Chopra, A.K., Penzien, J. (1980): Rocking response of rigid blocks to earthquakes. Earthquake Engng. Struct. Dyn., 8: 565-587. https://doi.org/10.1002/eqe.4290080606

[52] Agalianos, A., Psychari, A., Vassiliou, MF., Stojadinović, B., Anastasopoulos, I. (2017): Comparative assessment of two rocking isolation techniques for a motorway overpass bridge Front. Built Environ. https://doi.org/10.3389/fbuil.2017.00047

[53] Thomaidis, IM., Camara, A., Kappos, AJ. (2018): Simulating the rocking response of rigid bodies using general-purpose finite element software. $16^{\text {th }}$ European conference on Earthquake Engineering

[54] Pappas, A., Sextos, A., da Porto, F., Modena, C. (2017): Efficiency of alternative intensity measures for the seismic assessment of monolithic free-standing columns. Bull Earthquake Eng 15:1635-1659

[55] Sieber, M, Klar, S, Vassiliou, MF, Anastasopoulos, I. (2020) Robustness of simplified analysis methods for rocking structures on compliant soil. Earthquake Engng Struct Dyn. 2020; 49: 1388-1405. https://doi.org/10.1002/eqe.3294

[56] Vassiliou, MF, Broccardo, M, Cengiz, C, et al (2020): Shake table testing of a rocking podium: Results of a blind predictioncontest. Earthquake Engng Struct Dyn. 1- 20. https://doi.org/10.1002/eqe.3386

[57]Zhong, C, Christopoulos, C. (2020): Finite element analysis of the seismic shake-table response of a rocking podium structure. Earthquake Engng Struct Dyn. 1- 8. https://doi.org/10.1002/eqe.3397

[58] Malomo, D, Mehrotra, A, DeJong, MJ. (2020): Distinct element modeling of the dynamic response of a rocking podium tested on a shake table. Earthquake Engng Struct Dyn. 1-7. https://doi.org/10.1002/eqe.3404

[59] Vassiliou, MF., Cengiz, C., Dietz, M. et al. (2021) Dataset from shake table tests of free-standing rocking bodies. Earthquake Spectra (under review)

[60] Rezaeian S, Der Kiureghian A. (2008): A stochastic ground motion model with separable temporal and spectral nonstationarities. Earthq Eng Struct Dyn. 2008;37(13):1565-1584.

[61] Broccardo M, Dabaghi M. (2017) A spectral-based stochastic ground motion model with a non-parametric time-modulating function. In: $12^{\text {th }}$ International Conference on Structural Safety and Reliability; Vienna; 2017:1-10.

[62]ABAQUS 2019 (2019). Standard User's Manual. Providence, RI: Dassault Systèmes Simulia Corp.,

[63] Vassiliou, M. F., Mackie, K. R., and Stojadinović, B. (2017): A finite element model for seismic response analysis of deformable rocking frames. Earthquake Engng Struct. Dyn., 46: 447-466. doi: 10.1002/eqe.2799. 